\title{
Higher-Order Accelerations and Poles under the One-Parameter Planar Hyperbolic Motions and Their Inverse Motions
}

\author{
Serdal Şahin and Salim Yüce \\ Department of Mathematics, Faculty of Arts and Sciences, Yildiz Technical University, Esenler, 34210 Istanbul, Turkey \\ Correspondence should be addressed to Serdal Şahin; sersahin@yildiz.edu.tr
}

Received 23 May 2014; Accepted 19 September 2014; Published 12 October 2014

Academic Editor: Paulo Batista Gonçalves

Copyright (C) 2014 S. Şahin and S. Yüce. This is an open access article distributed under the Creative Commons Attribution License, which permits unrestricted use, distribution, and reproduction in any medium, provided the original work is properly cited.

\begin{abstract}
We expressed the higher-order velocities, accelerations, and poles under the one-parameter planar hyperbolic motions and their inverse motions. The higher-order accelerations and poles are also presented by considering the rotation angle $\varphi$ as a parameter of the motion and its inverse motion.
\end{abstract}

\section{Introduction}

First of all, we need to define the set of hyperbolic numbers. For this reason let us recall complex numbers, which are the extension of real numbers that include the imaginary unit $i$ and can be written in terms of the standard basis $\{1, i\}$ as $z=$ $x+i y$, where $x, y \in \mathbb{R}$. In this expression, $x$ is the real part and $y$ is the imaginary part of complex numbers.

Similarly, hyperbolic numbers can also be defined as an extension of the real numbers. This extension is performed by adjoining the unipotent (hyperbolic imaginary) $j$, where $j^{2}=1$ but $j \neq \pm 1$. In this case, the hyperbolic numbers set can be written as follows:

$$
\mathbb{H}=\mathbb{R}[j]=\left\{z=x+j y \mid x, y \in \mathbb{R}, j^{2}=1, j \neq \pm 1\right\} .
$$

Similar to complex numbers, hyperbolic numbers also have the standard basis $\{1, j\}$ and every hyperbolic number $z$ can be written in the form below:

$$
z=x+j y,
$$

where $x, y \in \mathbb{R}$. In this expression, the real and the unipotent parts are $x$ and $y$, respectively.

The collection of all hyperbolic numbers is called the hyperbolic plane $\mathbb{U}$ (or the split-complex plane). A complex number, $z \in \mathbb{C}$, is the point in the plane with the coordinates $(\operatorname{Re}(z), \operatorname{Im}(z))$. A hyperbolic number $z=x+j y$ can also be represented by the point $Z$ with the coordinates $(x, y)=$ $(\operatorname{Re}(z), \operatorname{Un}(z))$ and the conjugate of $z$ is defined by

$$
\bar{z}=x-j y .
$$

It has been useful to identify the hyperbolic numbers which have also been called perplex numbers, split-complex numbers, or double numbers with 2-dimensional Minkowski space-time. As the complex numbers are naturally related to the Euclidean plane, the hyperbolic numbers are related to the Lorentzian plane (or the 2-dimensional Minkowski spacetime).

Let $z=x+j y$ and $w=u+j v \in \mathbb{U}$; then the addition and multiplication of hyperbolic numbers can defined as follows:

$$
\begin{gathered}
z+w=(x+j y)+(u+j v)=(x+u)+j(y+v), \\
z \cdot w=(x+j y)(u+j v)=(x u+y v)+j(x v+y u) .
\end{gathered}
$$

This multiplication is commutative, associative, and distributive over addition.

Since the sets of hyperbolic numbers and complex numbers are two-dimensional vector spaces over the real numbers field, we can identify $z=x+j y$ with a point or vector $(x, y)$. The hyperbolic inner product is indicated as below:

$$
\langle z, w\rangle=\mathbb{R} e(z \bar{w})=\mathbb{R} e(\bar{z} w)=x u-y v,
$$


where $z=x+j y$ and $w=u+j v$. Hyperbolic numbers are said to be hyperbolic (Lorentzian) orthogonal if $\langle z, w\rangle=0$. The vectors are classified by the inner product as follows:

$$
\begin{aligned}
& \text { if }\langle z, z\rangle>0 \text { or } z=0, \text { then } z \text { is space-like; } \\
& \text { if }\langle z, z\rangle=0, \text { then } z \text { is null-like; } \\
& \text { if }\langle z, z\rangle<0, \quad \text { then } z \text { is time-like. }
\end{aligned}
$$

The hyperbolic modulus of $z=x+j y$ is defined by the following:

$$
\|z\|_{h}=\sqrt{|\langle z, z\rangle|}=\sqrt{|z \bar{z}|}=\sqrt{\left|x^{2}-y^{2}\right|}
$$

and is considered to be the hyperbolic distance to the point $z$ from the origin. Note that the points $z \neq 0$ on the lines $y= \pm x$ are isotropic because they are nonzero vectors with $\|z\|_{h}=0$. Thus, the hyperbolic distance yields a geometry, which is the Lorentzian geometry, on $\mathbb{R}^{2}$, quite unlike the usual Euclidean geometry of the complex plane, where $\|z\|_{h}=0$ only if $z=0$. Hyperbolic numbers are also the universal Clifford algebra for $\mathbb{R}^{1,0}$; see [1-4].

Every nonisotropic hyperbolic number $z=x+j y$ can be written in one of the forms below:

$$
z= \pm r e^{j \varphi}= \pm r(\cosh \varphi+j \sinh \varphi)
$$

or

$$
z= \pm r j e^{j \varphi}
$$

where $r=\sqrt{\left|x^{2}-y^{2}\right|}$ and $\varphi=\tanh ^{-1}(y / x)$ or $\varphi=$ $\tanh ^{-1}(x / y)$, respectively, which corresponds to 4 branches of the unit hyperbole $x^{2}-y^{2}= \pm 1$. In this case, the points of these branches $z= \pm j e^{j \varphi}$ and $z= \pm r j e^{j \varphi}$ are in quadrants,

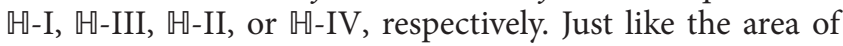
the region of the unit circle with a central angle $\phi$ is $(1 / 2) \phi$, the area of the unit hyperbolic region with a central angle $\varphi$ is also determined by $(1 / 2) \varphi$. A hyperbolic rotation by $e^{j \varphi}$ corresponds to the multiplication by the matrix

$$
\left[\begin{array}{ll}
\cosh \varphi & \sinh \varphi \\
\sinh \varphi & \cosh \varphi
\end{array}\right]
$$

Finally, if a vector is multiplied by $j$, it gives a hyperbolic orthogonal vector similar to multiplications by $i$ in the complex plane.

As seen above, the complex numbers plane and the hyperbolic numbers plane are quite similar. Hence, in analogy to one-parameter planar complex motion which was introduced by Blaschke and Müller [5], Yüce and Kuruoǧlu [6] also introduced the one-parameter planar hyperbolic motion and they obtained the velocities, accelerations, and poles accordingly.

\section{The Planar Hyperbolic Motion}

Let $\mathbb{H}$ and $\mathbb{H}^{\prime}$ be moving and fixed hyperbolic planes and let $\left\{O, \mathbf{h}_{\mathbf{1}}, \mathbf{h}_{\mathbf{2}}\right\}$ and $\left\{O^{\prime}, \mathbf{h}_{1}^{\prime}, \mathbf{h}_{2}^{\prime}\right\}$ be their orthonormal coordinate

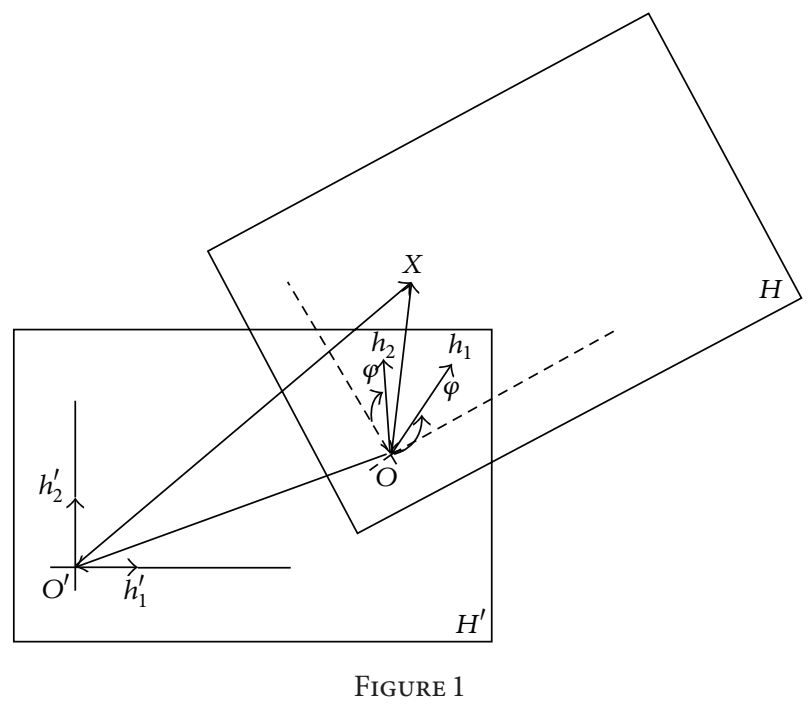

systems, respectively. If the vector $\overrightarrow{\mathrm{OO}^{\prime}}$ is represented by the hyperbolic number $\mathbf{u}^{\prime}$, then the motion can be defined with the aid of (8) and (10) by the transformation below:

$$
\mathbf{x}^{\prime}=\mathbf{u}^{\prime}+\mathbf{x} e^{j \varphi} .
$$

This transformation is called a one-parameter planar hyperbolic motion and denoted by $\mathbb{H} / \mathbb{H}^{\prime}$, where $\varphi$ is the rotation angle of the motion $\mathbb{H} / \mathbb{H}^{\prime}$; that is, the hyperbolic angle between the vectors $\mathbf{h}_{\mathbf{1}}$ and $\mathbf{h}_{\mathbf{1}}^{\prime}$ and the hyperbolic numbers $\mathbf{x}=x_{1}+j x_{2}$ and $\mathbf{x}^{\prime}=x_{1}^{\prime}+j x_{2}^{\prime}$ represent the point $X \in \mathbb{M}$ with respect to the moving and the fixed rectangular coordinate systems, respectively (Figure 1). Besides, the rotation angle $\varphi$ and $\mathbf{x}, \mathbf{x}^{\prime}$, and $\mathbf{u}^{\prime}$ are continuously differentiable functions of a time parameter $t \in \mathbb{\mathbb { R }}$ and at an initial time $t=0$, the coordinate systems are coincident.

Let the hyperbolic number $\mathbf{u}=u_{1}+j u_{2}$ represent the origin of the fixed point system with respect to the moving system. Then, if we take $X^{\prime}=O^{\prime}$, we obtain $\mathbf{x}^{\prime}=0$ and $\mathbf{x}=\mathbf{u}$. Thus, we can have $\mathbf{u}^{\prime}$ from (11):

$$
\mathbf{u}^{\prime}=-\mathbf{u} e^{j \varphi} .
$$

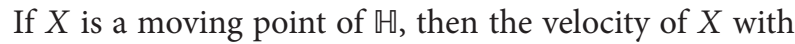
respect to $\mathbb{W}$ is known as the relative velocity of the motion $\mathbb{U} / \mathbb{U}^{\prime}$ and is denoted by $\mathbf{V}_{\mathbf{r}}$. This vector can be written as $\mathbf{V}_{\mathbf{r}}=$ $d \mathbf{x} / d t=\dot{\mathbf{x}}$. This vector can be expressed with respect to $\mathbb{U}^{\prime}$ by the equation below:

$$
\mathbf{V}_{\mathbf{r}}^{\prime}=\mathbf{V}_{\mathbf{r}} e^{j \varphi}=\dot{\mathbf{x}} e^{j \varphi}
$$

If we differentiate (11) with respect to $t$, we obtain the absolute velocity of the motion $\mathbb{U} / \mathbb{H}^{\prime}$ as follows:

$$
\mathbf{V}_{\mathbf{a}}^{\prime}=\frac{d \mathbf{x}^{\prime}}{d t}=\dot{\mathbf{x}}^{\prime}=\dot{\mathbf{u}}^{\prime}+(\dot{\mathbf{x}}+j \dot{\varphi} \mathbf{x}) e^{j \varphi},
$$

where

$$
\mathbf{V}_{\mathbf{f}}^{\prime}=\dot{\mathbf{u}}^{\prime}+j \dot{\varphi} \mathbf{x} e^{j \varphi}
$$


is the sliding velocity of the motion $\mathbb{H} / \mathbb{M}^{\prime}$. By differentiating (12) with respect to $t$, we also obtain the following equation:

$$
\dot{\mathbf{u}}^{\prime}=-(\dot{\mathbf{u}}+j \mathbf{u} \dot{\varphi}) e^{j \varphi} .
$$

Hence, we can rewrite the sliding velocity as follows:

$$
\mathbf{V}_{\mathbf{f}}^{\prime}=j \dot{\varphi} \mathbf{x} e^{j \varphi}-(\dot{\mathbf{u}}+j \mathbf{u} \dot{\varphi}) e^{j \varphi} .
$$

For a general one-parameter planar hyperbolic motion, there is a point that does not move, which means that its coordinates are the same in both reference coordinate systems $\left\{O ; \mathbf{e}_{1}, \mathbf{e}_{2}\right\}$ and $\left\{O^{\prime} ; \mathbf{e}_{1}^{\prime}, \mathbf{e}_{2}^{\prime}\right\}$. This point is called the pole point or the instantaneous rotation pole center. In this case, we obtain $\mathbf{V}_{\mathbf{f}}^{\prime}=\mathbf{0}$ and use (17); then, for the pole point $P^{\prime}=$ $p_{1}^{\prime}+j p_{2}^{\prime}$ of the motion, we get

$$
\mathbf{P}^{\prime}=p_{1}^{\prime}+j p_{2}^{\prime}=\mathbf{u}+j \frac{\dot{\mathbf{u}}}{\dot{\varphi}},
$$

so, from (17) and (18), we can rewrite the sliding velocity with the aid of the pole point as below:

$$
\mathbf{V}_{\mathbf{f}}^{\prime}=j \dot{\varphi}(\mathbf{x}-\mathbf{p}) e^{j \varphi} .
$$

During the motion $\mathbb{H} / \mathbb{U}^{\prime}$, the loci of the pole points $P \in$ $\mathbb{U}$, which are fixed on both planes for all $t \in \mathbb{\subset} \subset \mathbb{R}$, are called the moving and fixed pole curves and will be denoted by $(P)$ and $\left(P^{\prime}\right)$, respectively. Because the velocity of $(P)$ and $\left(P^{\prime}\right)$ is the same for each $t \in \mathbb{R},(P)$ and $\left(P^{\prime}\right)$ roll upon each other without sliding.

If we differentiate the relative velocity $\mathbf{V}_{\mathbf{f}}$ with respect to $t$, we obtain the relative acceleration vector $\mathbf{b}_{\mathbf{r}}$ as below:

$$
\mathbf{b}_{\mathbf{r}}=\dot{\mathbf{V}}_{\mathbf{r}}=\ddot{\mathbf{x}}
$$

This vector is expressed by the following:

$$
\mathbf{b}_{\mathbf{r}}^{\prime}=\mathbf{b}_{\mathbf{r}} e^{j \varphi}=\ddot{\mathbf{x}} e^{j \varphi}
$$

in the fixed coordinate system. During the motion $\mathbb{H} / \mathbb{H}^{\prime}$, if we differentiate the absolute velocity vector $\mathbf{V}_{\mathbf{a}}^{\prime}$ with respect to $t$, then we get the absolute acceleration vector $\mathbf{b}_{\mathbf{a}}^{\prime}$ as follows:

$$
\begin{aligned}
\mathbf{b}_{\mathbf{a}}^{\prime}= & \frac{d \mathbf{V}_{\mathbf{a}}^{\prime}}{d t} \\
= & \dot{\mathbf{V}}_{\mathbf{a}}^{\prime}=(\mathbf{x}-\mathbf{p})\left(j \ddot{\varphi}+\dot{\varphi}^{2}\right) e^{j \varphi} \\
& -j \dot{\varphi} \dot{\mathbf{p}} e^{j \varphi}+2 j \dot{\varphi} \dot{\mathbf{x}} e^{j \varphi}+\mathbf{b}_{\mathbf{r}}^{\prime},
\end{aligned}
$$

where

$$
\begin{aligned}
& \mathbf{b}_{\mathbf{f}}^{\prime}=(\mathbf{x}-\mathbf{p})\left(j \ddot{\varphi}+\dot{\varphi}^{2}\right) e^{j \varphi}-j \dot{\varphi} \dot{\mathbf{p}} e^{j \varphi}, \\
& \mathbf{b}_{\mathbf{c}}^{\prime}=2 j \dot{\varphi} \dot{\mathbf{x}} e^{j \varphi},
\end{aligned}
$$

are called the sliding acceleration vector and the coriolis acceleration vector of the motion $\mathbb{H} / \mathbb{H}^{\prime}$, respectively. From (22) and (23), we have the following equation:

$$
\mathbf{b}_{\mathrm{a}}^{\prime}=\mathbf{b}_{\mathrm{f}}^{\prime}+\mathbf{b}_{\mathrm{c}}^{\prime}+\mathbf{b}_{\mathrm{r}}^{\prime} \text {. }
$$

Under the motion $\mathbb{H} / \mathbb{H}^{\prime}$, the acceleration pole is characterized by eliminating the sliding acceleration vector $\mathbf{b}_{\mathrm{f}}^{\prime}$. Therefore, if we take $\mathbf{b}_{\mathbf{f}}^{\prime}=0$ and assume that $\ddot{\varphi}^{2}-\dot{\varphi}^{4} \neq 0$, then for the acceleration pole point $Q^{\prime}=\left(q_{1}^{\prime}, q_{2}^{\prime}\right) \in \mathbb{U}^{\prime}$ of the

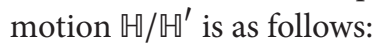

$$
\mathbf{p}^{\prime}=q_{1}^{\prime}+j q_{2}^{\prime}=\mathbf{p}+\frac{\dot{\varphi} \ddot{\varphi} \dot{\mathbf{p}}}{\ddot{\varphi}^{2}-\dot{\varphi}^{4}}-j \frac{\dot{\varphi}^{3} \dot{\mathbf{p}}}{\ddot{\varphi}^{2}-\dot{\varphi}^{4}} .
$$

\section{Higher-Order Accelerations and Poles under the One-Parameter Planar Hyperbolic Motions}

3.1. Higher-Order Accelerations under the One-Parameter Planar Hyperbolic Motions. Let the motion $\mathbb{H} / \mathbb{U}^{\prime}$ be the oneparameter planar hyperbolic motion and let $X \in \mathbb{U}$ be a fixed point. Then, the absolute velocity and the sliding velocity are equal to each other and this velocity is given as below:

$$
\mathbf{V}_{\mathbf{a}}^{\prime}=\mathbf{V}_{\mathbf{f}}^{\prime}=\dot{\mathbf{x}}^{\prime}=\dot{\mathbf{u}}^{\prime}+j \dot{\varphi}\left(\mathbf{x}^{\prime}-\mathbf{u}^{\prime}\right)
$$

If we differentiate (26) with respect to $t$, then we obtain the second-order velocity (absolute velocity or sliding velocity) as below:

$$
\ddot{\mathbf{x}^{\prime}}=\ddot{\mathbf{u}^{\prime}}+\left(j \ddot{\varphi}+\dot{\varphi}^{2}\right)\left(\mathbf{x}^{\prime}-\mathbf{u}^{\prime}\right) .
$$

From (26), let $\left(\mathbf{x}^{\prime}-\mathbf{u}^{\prime}\right)$ 's coefficient be $\Phi_{1}^{\prime}=j \dot{\varphi}$, and from (27), let $\left(\mathbf{x}^{\prime}-\mathbf{u}^{\prime}\right)$ 's coefficient be $\Phi_{2}^{\prime}=j \ddot{\varphi}+\dot{\varphi}^{2}$. Thus, we obtain $\Phi_{2}^{\prime}$ in terms of $\Phi_{1}^{\prime}$ as $\Phi_{2}^{\prime}=\dot{\Phi}_{1}^{\prime}+\Phi_{1}^{\prime} \Phi_{1}^{\prime}$. For this reason, from (26) and (27), we can rewrite $\dot{\mathbf{x}}^{\prime}$ and $\ddot{\mathbf{x}}^{\prime}$ as below:

$$
\begin{aligned}
& \dot{\mathbf{x}}^{\prime}=\dot{\mathbf{u}}^{\prime}+\Phi_{1}^{\prime}\left(\mathbf{x}^{\prime}-\mathbf{u}^{\prime}\right), \\
& \ddot{\mathbf{x}^{\prime}}=\ddot{\mathbf{u}}^{\prime}+\Phi_{2}^{\prime}\left(\mathbf{x}^{\prime}-\mathbf{u}^{\prime}\right) .
\end{aligned}
$$

In this way, if we differentiate (29) with respect to $t$, then we obtain the third-order velocity (or the second-order acceleration) as below:

$$
\ddot{\mathbf{x}^{\prime}}=\ddot{\mathbf{u}^{\prime}}+\dot{\Phi}_{2}^{\prime}\left(\mathbf{x}^{\prime}-\mathbf{u}^{\prime}\right)+\Phi_{2}^{\prime}\left(\dot{\mathbf{x}^{\prime}}-\dot{\mathbf{u}}^{\prime}\right)
$$

If we use (28) in the latter equation, we have the following:

$$
\mathbf{x}^{\prime}=\ddot{\mathbf{u}}^{\prime}+\left(\dot{\Phi}_{2}^{\prime}+\Phi_{2}^{\prime} \Phi_{1}^{\prime}\right)+\left(\mathbf{x}^{\prime}-\mathbf{u}^{\prime}\right)
$$

or

$$
\mathbf{x}^{\prime}=\mathbf{u}^{\prime}+\Phi_{3}^{\prime}+\left(\mathbf{x}^{\prime}-\mathbf{u}^{\prime}\right)
$$

where $\Phi_{3}^{\prime}=\dot{\Phi}_{2}^{\prime}+\Phi_{2}^{\prime} \Phi_{1}^{\prime} \neq 0$. If we differentiate (32) with respect to $t$, then we obtain the fourth-order velocity (or the third-order acceleration) as below:

$$
\stackrel{(4)}{\mathbf{x}^{\prime}}=\stackrel{(4)}{\mathbf{u}^{\prime}}+\dot{\Phi_{3}^{\prime}}\left(\mathbf{x}^{\prime}-\mathbf{u}^{\prime}\right)+\Phi_{3}^{\prime}\left(\dot{\mathbf{x}^{\prime}}-\dot{\mathbf{u}^{\prime}}\right)
$$


So, from (28) and the latter equation, we have the following:

$$
\mathbf{x}^{\prime}=\stackrel{(4)}{\mathbf{u}^{\prime}}+\left(\dot{\Phi_{3}^{\prime}}+\Phi_{3}^{\prime} \Phi_{1}^{\prime}\right)+\left(\mathbf{x}^{\prime}-\mathbf{u}^{\prime}\right)
$$

or

$$
\stackrel{(4)}{\mathbf{x}^{\prime}}=\stackrel{(4)}{\mathbf{u}^{\prime}}+\Phi_{4}^{\prime}+\left(\mathbf{x}^{\prime}-\mathbf{u}^{\prime}\right)
$$

where $\Phi_{4}^{\prime}=\dot{\Phi}_{3}^{\prime}+\Phi_{3}^{\prime} \Phi_{1}^{\prime} \neq 0$.

If we continue to subsequent differentiations, we can get higher-order velocities and accelerations. As we can clearly see in the following equations,

$$
\begin{gathered}
\stackrel{(n)}{\prime \prime}=\stackrel{(n)}{\mathbf{x}^{\prime}}=\mathbf{x}^{\prime}+\Phi_{n}^{\prime}\left(\mathbf{x}^{\prime}-\mathbf{u}^{\prime}\right), \\
\Phi_{n}^{\prime}=\dot{\Phi}_{(n-1)}^{\prime}+\Phi_{(n-1)}^{\prime} \Phi_{1}^{\prime} \neq 0,
\end{gathered}
$$

are true for $n=1, n=2, n=3$, and $n=4$ (where we suppose that $\left.\Phi_{0}^{\prime}=1\right)$. If we assume that (36) and (37) are also true, then we can get the following:

$$
\stackrel{(n+1)}{\mathbf{x}^{\prime}}=\stackrel{(n+1)}{\mathbf{u}^{\prime}}+\left(\dot{\Phi}_{n}^{\prime}+\Phi_{n}^{\prime} \Phi_{1}^{\prime}\right)\left(\mathbf{x}^{\prime}-\mathbf{u}^{\prime}\right)
$$

by differentiation (36). Furthermore, we can write $\Phi_{(n+1)}^{\prime}=$ $\dot{\Phi}_{n}^{\prime}+\Phi_{n}^{\prime} \Phi_{1}^{\prime} \neq 0$ from (37). Finally, (36) has been proved by the induction method. Thus, we may give the following theorem.

Theorem 1. During the one-parameter planar hyperbolic motion $\mathbb{H} / \mathbb{H}^{\prime}$, if $X \in \mathbb{H}$ is a fixed point, then we get

$$
\stackrel{(n)}{\mathbf{x}^{\prime}}=\stackrel{(n)}{\mathbf{u}^{\prime}}+\Phi_{n}^{\prime}\left(\mathbf{x}^{\prime}-\mathbf{u}^{\prime}\right)
$$

for the velocities from $n$ th-order and the acceleration from ( $n-$ 1) th order for each $t$, where

$$
\begin{gathered}
\Phi_{1}^{\prime}=j \dot{\varphi}, \quad \Phi_{0}^{\prime}=1, \quad \stackrel{(0)}{\mathbf{x}^{\prime}}=\mathbf{x}^{\prime}, \\
\Phi_{n}^{\prime}=\dot{\Phi}_{(n-1)}^{\prime}+\Phi_{n-1}^{\prime} \Phi_{1}^{\prime} \neq 0 .
\end{gathered}
$$

3.2. Higher-Order Poles under the One-Parameter Planar Hyperbolic Motions. During the one-parameter planar hyperbolic motion $\mathbb{T} / \mathbb{W}^{\prime}$, if $X \in \mathbb{M}$ is a fixed point, then from (28), we can calculate the first-order pole point as follows:

$$
\mathbf{x}^{\prime}=\mathbf{p}_{1}^{\prime}=\mathbf{u}^{\prime}-\frac{\dot{\mathbf{u}^{\prime}}}{\Phi_{1}^{\prime}}
$$

By using (29), we obtain the second-order pole point as below:

$$
\mathbf{x}^{\prime}=\mathbf{p}_{2}^{\prime}=\mathbf{u}^{\prime}-\frac{\ddot{\mathbf{u}}^{\prime}}{\Phi_{2}^{\prime}} .
$$

In a similar way, from (37), we can get the $(n-1)$ th-order acceleration pole as shown below:

$$
\mathbf{x}^{\prime}=\mathbf{p}_{n}^{\prime}=\mathbf{u}^{\prime}-\frac{(n)}{\mathbf{u}^{\prime}}
$$

Also, from (39), we have the following:

$$
\mathbf{u}^{\prime}=\Phi_{n}^{\prime}\left(\mathbf{u}^{\prime}-\mathbf{p}_{n}^{\prime}\right)
$$

So, if we use (37) and the latter equation, we can rewrite $\mathbf{x}^{\prime}$ in terms of $\mathbf{p}_{n}^{\prime}$ as follows:

$$
\stackrel{(n)}{\mathbf{x}^{\prime}}=\Phi_{n}^{\prime}\left(\mathbf{x}^{\prime}-\mathbf{p}_{n}^{\prime}\right)
$$

Similarly, we can obtain the higher-order pole points with respect to moving coordinate system $\mathbb{H}$ as follows:

$$
\mathbf{x}=\mathbf{p}_{n}=-\frac{\mathbf{u}^{\prime}}{\Phi_{n}^{\prime}} e^{-j \varphi}
$$

If the absolute value of both sides of (45) is taken, then one can find that the hyperbolic number $\Phi_{n}^{\prime}$ is independent from the point $X$. So we can give the following theorems.

Theorem 2. The absolute value of higher-order accelerations under the one-parameter planar hyperbolic motion is rational with distance between the point $X$ and corresponding pole point and it takes the same value above the circles which have this pole point as a center.

Theorem 3. If we consider higher-order acceleration vectors $\mathbf{x}^{\prime}=\Phi_{n}^{\prime}\left(\mathbf{x}^{\prime}-\mathbf{p}_{n}^{\prime}\right)$ corresponding order pole rays $\mathbf{P}_{n}^{\prime} \mathbf{X}^{\prime}$ of all points $X$ of the moving plane for each $t$, we can obtain the same angle between these vectors $\psi=\operatorname{arctanh}\left(\operatorname{Un}\left(\Phi_{n}^{\prime}\right) / \operatorname{Re}\left(\Phi_{n}^{\prime}\right)\right)$ in the case of both of the space-like and time-like vectors and we can also obtain the same angle between these vectors $\psi=$ $\operatorname{arctanh}\left(\operatorname{Re}\left(\Phi_{n}^{\prime}\right) / \mathrm{Un}\left(\Phi_{n}^{\prime}\right)\right)$ in case one of these vectors is spacelike and the other is time-like.

For more properties of hyperbolic angle, the reader is referred to [7-9].

\section{The Inverse Motion $\mathbb{W}^{\prime} / \mathbb{H}$ of the One- Parameter Planar Hyperbolic Motion $\mathbb{U} / \mathbb{T}^{\prime}$ and Higher-Order Accelerations and Poles under This Motion}

Let $\mathbb{H}$ and $\mathbb{H}^{\prime}$ be fixed and moving hyperbolic planes and $\left\{O, \mathbf{h}_{\mathbf{1}}, \mathbf{h}_{\mathbf{2}}\right\}$ and $\left\{O^{\prime}, \mathbf{h}_{\mathbf{1}}^{\prime}, \mathbf{h}_{2}^{\prime}\right\}$ their orthonormal coordinate systems, respectively. Then we obtain the inverse motion of the motion $\mathbb{H} / \mathbb{H}^{\prime}$, denoted by $\mathbb{H}^{\prime} / \mathbb{H}$. In this motion, all of the velocities, accelerations, and poles are analyzed in $\mathbb{H}$, which is the fixed plane during the inverse motion of the oneparameter planar hyperbolic motion, instead of $\mathbb{H}^{\prime}$. This is the main difference from the one-parameter planar hyperbolic motion. So, from (11), we can obtain the transformation of the one-parameter planar inverse hyperbolic motion $\mathbb{H}^{\prime} / \mathbb{H}$ with the below solution way:

$$
\mathbf{x}=\left(\mathbf{x}^{\prime}-\mathbf{u}^{\prime}\right) e^{-j \varphi}
$$


and form (12) can be written as follows:

$$
\mathbf{x}=\mathbf{u}+\mathbf{x}^{\prime} e^{-j \varphi}
$$

where $-\varphi$ is the rotation angle of the motion $\mathbb{H}^{\prime} / \mathbb{H}$. In analogy to the motion $\mathbb{H} / \mathbb{U}^{\prime}$, we can get the velocities, accelerations, and poles for the motion $\mathbb{H}^{\prime} / \mathbb{H}$. But we want to focus on higher-order accelerations and poles.

4.1. Higher-Order Accelerations under the One-Parameter Planar Inverse Hyperbolic Motions. During the motion $\mathbb{U}^{\prime} / \mathbb{H}$, $X^{\prime} \in \mathbb{H}^{\prime}$ is a fixed point, then the absolute velocity and the sliding velocity are equal to each other, and this velocity is given as below:

$$
\dot{\mathbf{x}}=\dot{\mathbf{u}}-j \dot{\varphi} \mathbf{x}^{\prime} e^{-j \varphi}
$$

It is convenient to write the latter equation as follows:

$$
\dot{\mathbf{x}}=\dot{\mathbf{u}}-j \dot{\varphi}(\mathbf{x}-\mathbf{u}) .
$$

If we differentiate (50) with respect to $t$, then we obtain the second-order velocity (absolute velocity or sliding velocity) as below:

$$
\ddot{\mathbf{x}}=\ddot{\mathbf{u}}-j \ddot{\varphi}(\mathbf{x}-\mathbf{u})-j \dot{\varphi}(\dot{\mathbf{x}}-\dot{\mathbf{u}})
$$

or from (50) and the latter equation, we obtain the secondorder velocity (or the first-order acceleration) as below:

$$
\ddot{\mathbf{x}}=\ddot{\mathbf{u}}-\left(j \ddot{\varphi}-\dot{\varphi}^{2}\right)(\mathbf{x}-\mathbf{u}) .
$$

From (50) and (52), we can write that the coefficients of $(\mathbf{x}-\mathbf{u})$ are $\Phi_{1}=-j \dot{\varphi}$ and $\Phi_{2}=-j \ddot{\varphi}+\dot{\varphi}^{2}$, respectively. So we can obtain $\Phi_{2}$ in terms of $\Phi_{1}$ as $\Phi_{2}=\dot{\Phi}_{1}+\Phi_{1} \Phi_{1}$. It is convenient to rewrite (50) and (52) as below:

$$
\begin{aligned}
& \dot{\mathbf{x}}=\dot{\mathbf{u}}+\Phi_{1}(\mathbf{x}-\mathbf{u}), \\
& \ddot{\mathbf{x}}=\ddot{\mathbf{u}}+\Phi_{2}(\mathbf{x}-\mathbf{u}) .
\end{aligned}
$$

Similarly, if we differentiate (54) with respect to $t$, then we obtain the third-order velocity (or the second-order acceleration) such as

$$
\dddot{\mathbf{x}}=\dddot{\mathbf{u}}+\dot{\Phi}_{2}(\mathbf{x}-\mathbf{u})+\Phi_{2}(\dot{\mathbf{x}}-\dot{\mathbf{u}}) .
$$

If we use (50) in the latter equation, we get one of the following equations:

$$
\dddot{\mathbf{x}}=\dddot{\mathbf{u}}+\left(\dot{\Phi}_{2}+\Phi_{2} \Phi_{1}\right)(\mathbf{x}-\mathbf{u}),
$$

or

$$
\dddot{\mathbf{x}}=\dddot{\mathbf{u}}+\Phi_{3}(\mathbf{x}-\mathbf{u})
$$

where $\Phi_{3}=\dot{\Phi}_{2}+\Phi_{2} \Phi_{1} \neq 0$. If we differentiate (57) with respect to $t$, then we obtain the fourth-order velocity (or the third-order acceleration) as below:

$$
\stackrel{(4)}{\mathbf{x}}=\stackrel{(4)}{\mathbf{u}}+\dot{\Phi}_{3}(\mathbf{x}-\mathbf{u})+\Phi_{3}(\dot{\mathbf{x}}-\dot{\mathbf{u}}) .
$$

So, from (50) and the latter equation, we have the following:

$$
\stackrel{(4)}{\mathbf{x}}=\stackrel{(4)}{\mathbf{u}}+\left(\dot{\Phi}_{3}+\Phi_{3} \Phi_{1}\right)(\mathbf{x}-\mathbf{u})
$$

or

$$
\stackrel{(4)}{\mathbf{x}}=\stackrel{(4)}{\mathbf{u}}+\Phi_{4}(\mathbf{x}-\mathbf{u}),
$$

where $\Phi_{4}=\dot{\Phi}_{3}+\Phi_{3} \Phi_{1} \neq 0$.

If we continue to subsequent differentiations, we can get the higher-order velocities and accelerations. So, we can clearly see that the following equations

$$
\begin{gathered}
\stackrel{(n)}{\mathbf{x}}=\stackrel{(n)}{\mathbf{u}}+\Phi_{n}^{\prime}\left(\mathbf{x}^{\prime}-\mathbf{u}^{\prime}\right), \\
\Phi_{n}=\dot{\Phi}_{(n-1)}+\Phi_{(n-1)} \Phi_{1} \neq 0
\end{gathered}
$$

are true for $n=1, n=2, n=3$, and $n=4$ (we suppose that $\left.\Phi_{0}=1\right)$. If we assume that (61) and (62) are also true, then we can get the following:

$$
\stackrel{(n+1)}{\mathbf{x}}=\stackrel{(n+1)}{\mathbf{u}}+\left(\dot{\Phi}_{n}+\Phi_{n} \Phi_{1}\right)(\mathbf{x}-\mathbf{u}),
$$

by differentiation (61). Furthermore, we can write

$$
\Phi_{(n+1)}=\dot{\Phi}_{n}+\Phi_{n} \Phi_{1} \neq 0
$$

from (62). Finally, (61) has been proved by the induction method. Thus, we may give the following theorem.

Theorem 4. During the one-parameter planar inverse hyperbolic motion $\mathbb{H}^{\prime} / \mathbb{U}$, if $X^{\prime} \in \mathbb{H}^{\prime}$ is a fixed point, then we get

$$
\stackrel{(n)}{\mathbf{x}}=\stackrel{(n)}{\mathbf{u}}+\Phi_{n}(\mathbf{x}-\mathbf{u}),
$$

for the velocities from $n$ th-order and the acceleration from ( $n-$ 1) th-order for each $t$, where

$$
\begin{gathered}
\Phi_{1}=-j \dot{\varphi}, \quad \Phi_{0}=1, \quad \stackrel{(0)}{\mathbf{x}}=\mathbf{x}, \\
\Phi_{n}=\dot{\Phi}_{n-1}+\Phi_{n-1} \Phi_{1} \neq 0 .
\end{gathered}
$$

4.2. Higher-Order Poles under the One-Parameter Planar Inverse Hyperbolic Motion. During the one-parameter planar inverse hyperbolic motion $\mathbb{H}^{\prime} / \mathbb{W}$, from (50), we can calculate the first-order pole point for the fixed point $X^{\prime} \in \mathbb{H}^{\prime}$ as below:

$$
\mathbf{x}=\mathbf{q}_{1}=\mathbf{u}-\frac{\dot{\mathbf{u}}}{\Phi_{1}} .
$$

By using (54), we obtain the second-order pole point as below:

$$
\mathbf{x}=\mathbf{q}_{2}=\mathbf{u}-\frac{\ddot{\mathbf{u}}}{\Phi_{2}} .
$$

In a similar way, from (57), we can get the $(n-1)$ th-order acceleration pole as follows:

$$
\mathbf{x}=\mathbf{q}_{n}=\mathbf{u}-\frac{\stackrel{(n)}{\mathbf{u}}}{\Phi_{n}} .
$$


Also, from (70), we can write the following equation:

$$
\stackrel{(n)}{\mathbf{u}}=\Phi_{n}\left(\mathbf{u}-\mathbf{q}_{n}\right)
$$

So, if we use (65) and the latter equation, we can rewrite ${ }^{(n)} \mathbf{x}$ in terms of $\mathbf{q}_{n}$ as follows:

$$
\stackrel{(n)}{\mathbf{x}}=\Phi_{n}\left(\mathbf{x}-\mathbf{q}_{n}\right)
$$

Similarly, we can obtain the higher-order pole points under the one-parameter planar inverse hyperbolic motion $\mathbb{H}^{\prime} / \mathbb{H}$, with respect to moving coordinate system $\mathbb{U}^{\prime}$ as follows:

$$
\mathbf{x}^{\prime}=\mathbf{q}_{n}^{\prime}=-\frac{(n)}{\Phi_{n}} e^{j \varphi}
$$

\section{The Relationships between $\mathbb{H} / \mathbb{T}^{\prime}$ and $\mathbb{U}^{\prime} / \mathbb{W}$}

5.1. The Relationship between Functions $\Phi_{n}^{\prime}$ and $\Phi_{n}$. We are going to obtain two relationships between functions $\Phi_{n}^{\prime}$ and $\Phi_{n}$, during the motions $\mathbb{H} / \mathbb{W}^{\prime}$ and $\mathbb{H}^{\prime} / \mathbb{H}$. We are going to use the induction method for the first one. For this reason, we can see that the following equation

$$
\Phi_{n}=\overline{\Phi_{n}^{\prime}}, \quad \Phi_{n}^{\prime}=\overline{\Phi_{n}},
$$

is true for $n=1, n=2$, and $n=3$. Now, let us assume that (74) is true for $n=k$ and prove that it is true for $n=k+1$. Hence, if we assume that $\Phi_{k}=\overline{\Phi_{k}^{\prime}}$ is true, from (67) we can obtain the following:

$$
\Phi_{k+1}=\dot{\Phi}_{k}+\Phi_{k} \Phi_{1}
$$

Then, because of $\Phi_{k}=\overline{\Phi_{k}^{\prime}}$ and $\Phi_{n}^{\prime}=\overline{\Phi_{n}}$ we get the below equation:

$$
\Phi_{k+1}=\overline{\dot{\Phi}_{k}^{\prime}}+\overline{\Phi_{k}^{\prime}} \overline{\Phi_{1}^{\prime}}=\overline{\Phi_{k+1}^{\prime}}
$$

So, we can give the following theorem.

Theorem 5. During the motions $\mathbb{U} / \mathbb{U}^{\prime}$ and $\mathbb{M} / \mathbb{M}$, the following relationship between functions $\Phi_{n}^{\prime}$ and $\Phi_{n}$

$$
\Phi_{n}=\overline{\Phi_{n}^{\prime}}, \quad \Phi_{n}^{\prime}=\overline{\Phi_{n}},
$$

holds.

For the other relationship between functions $\Phi_{n}^{\prime}$ and $\Phi_{n}$, if we differentiate functions $e^{j \varphi}$ and $e^{-j \varphi}$ with respect to $t$, we can get the following:

$$
\begin{gathered}
\frac{d}{d t} e^{j \varphi}=\Phi_{1}^{\prime} e^{j \varphi}, \\
\frac{d^{2}}{d t^{2}} e^{j \varphi}=\left(\dot{\Phi}_{1}^{\prime}+\Phi_{1}^{\prime 2}\right) e^{j \varphi}=\Phi_{2}^{\prime} e^{j \varphi} .
\end{gathered}
$$

If we continue with consecutive differentiations, we obtain the following:

$$
\frac{d^{n}}{d t^{n}} e^{j \varphi}=\Phi_{n}^{\prime} e^{j \varphi}
$$

or

$$
\Phi_{n}^{\prime}=e^{-j \varphi} \frac{d^{n}}{d t^{n}} e^{j \varphi}
$$

Similarly, we get the following:

$$
\Phi_{n}=e^{j \varphi} \frac{d^{n}}{d t^{n}} e^{-j \varphi}
$$

From $\left(d^{n} / d t^{n}\right)\left(e^{j \varphi} e^{-j \varphi}\right)=0$ and Leibniz formula $\left(\left(d^{n} / d t^{n}\right)(u, v)=\sum_{k=0}^{n}\left(\begin{array}{l}n \\ k\end{array}\right) \stackrel{(k)}{u} \stackrel{(n-k)}{v}\right)$, we can give the following theorem.

Theorem 6. During the motions $\mathbb{H} / \mathbb{U}^{\prime}$ and $\mathbb{H}^{\prime} / \mathbb{H}$, the following relationship between functions $\Phi_{n}^{\prime}$ and $\Phi_{n}$

$$
\sum_{k=0}^{n}\left(\begin{array}{l}
n \\
k
\end{array}\right) \Phi_{k}^{\prime} \Phi_{n-k}=\sum_{k=0}^{n}\left(\begin{array}{l}
n \\
k
\end{array}\right) \Phi_{k} \Phi_{n-k}^{\prime}=0
$$

holds.

5.2. The Relationship between Poles $\mathbf{P}_{n}$ and $\mathbf{Q}_{n}$. From (43), (46), (70), and (73), we can rewrite the $\mathbf{p}_{n}^{\prime}, \mathbf{p}_{n}$ and $\mathbf{q}_{n}, \mathbf{q}_{n}^{\prime}$ as follows:
(i) $\mathbf{x}^{\prime}=\mathbf{p}_{n}^{\prime}=\mathbf{u}^{\prime}-\frac{(n)}{\mathbf{u}^{\prime}}$,
(ii) $\mathbf{x}=\mathbf{p}_{n}=-\frac{(n)}{\Phi_{n}^{\prime}} e^{-j \varphi}$,
(i) $\mathbf{x}=\mathbf{q}_{n}=\mathbf{u}-\frac{\stackrel{(n)}{\mathbf{u}}}{\Phi_{n}}$,
(ii) $\mathbf{x}^{\prime}=\mathbf{q}_{n}^{\prime}=-\frac{(n)}{\Phi_{n}} e^{j \varphi}$.

Furthermore, we can write the $n$ th-order derivative of (12) as below:

$$
\stackrel{(n)}{\mathbf{u}^{\prime}}=-\sum_{k=0}^{n}\left(\begin{array}{l}
n \\
k
\end{array}\right) \stackrel{(n)}{\mathbf{u}} \Phi_{n-k}^{\prime} e^{j \varphi},
$$

with the aid of (80) and Leibniz formula. Then, from (84) part (ii), we can obtain $\stackrel{(n)}{\mathbf{u}}=-\mathbf{q}_{n}^{\prime} \Phi_{n} e^{-j \varphi}$. Also, if we use this $(n)$

expression we can rewrite $\mathbf{u}^{\prime}$ as follows:

$$
\stackrel{(n)}{\mathbf{u}^{\prime}}=\sum_{k=0}^{n}\left(\begin{array}{l}
n \\
k
\end{array}\right) \mathbf{q}_{k}^{\prime} \Phi_{k} \Phi_{n-k}^{\prime} .
$$

We know this from (84) $\mathbf{q}_{0}=0$ and $\mathbf{q}_{0}^{\prime}=\mathbf{u}^{\prime}$. Hence, we can write another expression for $\mathbf{u}^{\prime}$ as follows:

$$
\mathbf{u}^{\prime}=\mathbf{u}^{\prime} \Phi_{n}^{\prime}+\sum_{k=1}^{n}\left(\begin{array}{l}
n \\
k
\end{array}\right) \mathbf{q}_{k}^{\prime} \Phi_{k} \Phi_{n-k}^{\prime}
$$

Ultimately, if we use (87) and (83) part (i), we can give the following theorem. 
Theorem 7. During the motions $\mathbb{H} / \mathbb{U}^{\prime}$ and $\mathbb{U}^{\prime} / \mathbb{M}$, the following equation between $\mathbf{p}_{n}^{\prime}$ and $\mathbf{q}_{n}^{\prime}$

$$
\mathbf{p}_{n}^{\prime}=-\frac{1}{\Phi_{n}^{\prime}} \sum_{k=1}^{n}\left(\begin{array}{l}
n \\
k
\end{array}\right) \mathbf{q}_{k}^{\prime} \Phi_{k} \Phi_{n-k}^{\prime}
$$

holds.

Similarly, we can obtain the following results:

Result 1

$$
\mathbf{q}_{n}^{\prime}=-\frac{1}{\Phi_{n}^{\prime}} \sum_{k=1}^{n}\left(\begin{array}{l}
n \\
k
\end{array}\right) \mathbf{p}_{k}^{\prime} \Phi_{k}^{\prime} \Phi_{n-k} .
$$

Result 2

$$
\mathbf{p}_{n}=-\frac{1}{\Phi_{n}^{\prime}} \sum_{k=1}^{n}\left(\begin{array}{l}
n \\
k
\end{array}\right) \mathbf{q}_{k} \Phi_{k} \Phi_{n-k}^{\prime} .
$$

Result 3

$$
\mathbf{q}_{n}=-\frac{1}{\Phi_{n}^{\prime}} \sum_{k=1}^{n}\left(\begin{array}{l}
n \\
k
\end{array}\right) \mathbf{p}_{k} \Phi_{k}^{\prime} \Phi_{n-k} .
$$

\section{Rotation Angle as a Parameter}

In this section, we choose the rotation angle $\varphi$ as a parameter of the motion $\mathbb{H} / \mathbb{H}^{\prime}$ instead of the parameter $t$. The purpose of this choice is to get the formulation in Sections 2, 3, and 4 that are more simple. So, if we use the rotation angle $\varphi$ as a parameter, then we obtain $\Phi_{n}^{\prime}$ and $\Phi_{n}$ as follows:

$$
\Phi_{n}^{\prime}=j^{n}, \quad \Phi_{n}=(-j)^{n} .
$$

Now, with the aid of the last equation, we can rewrite (40) and (41) as below:

$$
\begin{gathered}
\mathbf{p}_{n}^{\prime}=\mathbf{u}^{\prime}-\frac{\mathbf{u}^{\prime}}{j^{n}}=\mathbf{u}^{\prime}-j^{-n} \stackrel{(n)}{\mathbf{u}^{\prime},} \\
(n) \\
\mathbf{x}^{\prime}=j^{n}\left(\mathbf{x}^{\prime}-\mathbf{p}_{n}^{\prime}\right) .
\end{gathered}
$$

Furthermore, from (94), we can write $\mathbf{p}_{n}^{\prime}$ as below:

$$
\mathbf{p}_{n}^{\prime}=\mathbf{x}^{\prime}-j^{-n} \stackrel{(n)}{\mathbf{x}^{\prime}}
$$

Similarly, from (72), we can obtain $\mathbf{x}_{n}$ and $\mathbf{q}_{n}$ as below:

$$
\begin{gathered}
\stackrel{(n)}{\mathbf{x}}=(-j)^{n}\left(\mathbf{x}-\mathbf{q}_{n}\right), \\
\mathbf{q}_{n}=\mathbf{x}-j^{-n} \stackrel{(n)}{\mathbf{x}} .
\end{gathered}
$$

Hence, it is known that the multiplication with a hyperbolic number like $z=x+j y$ means rotating as its argument and translates as its module to the multiplicand vector. But the isotropic lines divide the hyperbolic plane into four separate

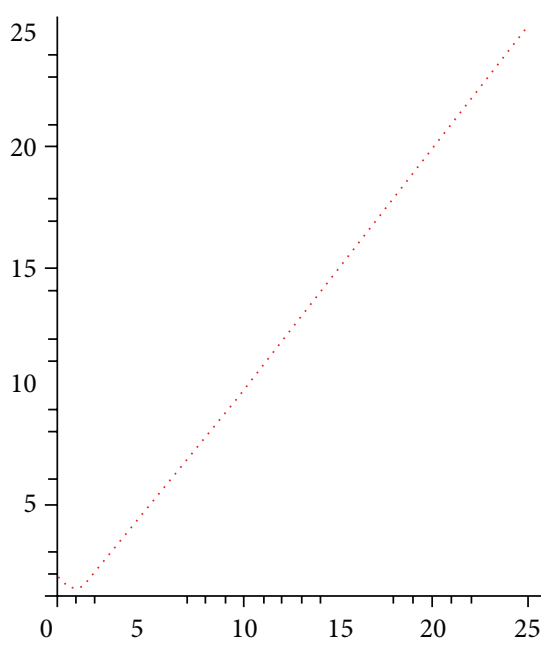

FIGURE 2

regions because of the fact that we must be careful if the multiplication with hyperbolic numbers changes the region or not. For instance, if we multiplicate $z_{1}=x_{1}+j y_{1} \in \mathbb{H}-\mathrm{I}$ and $z_{2}=x_{2}+j y_{2} \in \mathbb{M}$-II, we obtain $z=z_{1} z_{2} \in \mathbb{M}$-II, where

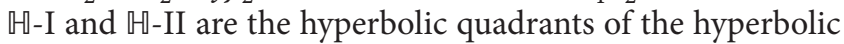
plane; see [10]. Hence, we can give the following theorem.

Theorem 8. During the one-parameter planar hyperbolic motion $\mathbb{H} / \mathbb{H}^{\prime}$, if we choose the rotation angle $\varphi$ as a parameter of the motion instead of the parameter $t$, then adding $\mathbf{x}^{\prime}$ to the vector, which we have obtained by multiplication with the hyperbolic number $z=-j^{-n}$ and $\mathbf{x}^{\prime}$, is giving the nth-order pole point (or $(n-1)$ th-order acceleration pole point) of the planar hyperbolic motion, $\mathbf{p}_{n}^{\prime}$. Also, we add $\mathbf{x}$ to the vector which we have obtained by multiplication with the hyperbolic number $z=-j^{-n}$ and ${ }^{(n)} \mathbf{x}$ is giving the $n$ th-order pole point (or $(n-1)$ thorder acceleration pole point) of the inverse planar hyperbolic motion, $\mathbf{q}_{n}$.

Example 9. Let us consider the one-parameter planar hyperbolic motion with $u(t)=\cos (t)+j \sin (t)$ and $\varphi(t)=(\pi / 6) t$. In this situation we can show the curve of the point $z=1+j 2 \epsilon$ $\mathbb{W}$ by using Maple Programming for $t \in[0,5]$ on the plane $\mathbb{U}^{\prime}$ (Figure 2).

Then, we calculate by using Maple Programming also the higher-order accelerations of the point $z=1+j 2 \in \mathbb{H}$ under this motion for $n=1,2,3$ as follows:

$$
\begin{aligned}
\mathbf{x}^{\prime}=e^{j(\pi t / 6)}[ & \left(\sin (t)-\frac{\pi}{6} \sin (t)+\frac{\pi}{3}\right) \\
& \left.+j\left(-\cos (t)-\frac{\pi}{6} \cos (t)+\frac{\pi}{6}\right)\right], \\
\mathbf{x}^{\prime}=e^{j(\pi t / 6)}[ & \left(\cos (t)-\frac{\pi}{3} \cos (t)-\frac{\pi^{2}}{36} \cos (t)+\frac{\pi^{2}}{36}\right) \\
& \left.+j\left(\sin (t)+\frac{\pi}{3} \sin (t)-\frac{\pi^{2}}{36} \sin (t)+\frac{\pi^{2}}{18}\right)\right],
\end{aligned}
$$




$$
\begin{gathered}
\mathbf{x}^{\prime}=e^{j(\pi t / 6)} \\
\times\left[\left(-\sin (t)+\frac{\pi}{2} \sin (t)+\frac{\pi^{2}}{12} \sin (t)\right.\right. \\
\left.-\frac{\pi^{3}}{216} \sin (t)+\frac{\pi^{3}}{108}\right) \\
+j\left(\cos (t)+\frac{\pi}{2} \cos (t)-\frac{\pi^{2}}{12} \cos (t)\right. \\
\left.\left.-\frac{\pi^{3}}{216} \cos (t)+\frac{\pi^{3}}{216}\right)\right],
\end{gathered}
$$

where $e^{j(\pi t / 6)}=\cosh (\pi t / 6)+j \sin (\pi t / 6)$. Similarly, we can calculate the higher-order poles of the point $z=1+j 2 \in \mathbb{M}$ under this motion for $n=1,2,3$ as follows:

$$
\begin{aligned}
\mathbf{p}_{1}^{\prime}= & -u e^{j(\pi t / 6)} \\
& -\frac{6 e^{j(\pi t / 6)}(\sin (t)-j \cos (t)-(\pi / 6)(j \cos t+\sin t))}{j \pi}, \\
\mathbf{p}_{2}^{\prime}= & -u e^{j(\pi t / 6)} \\
& -\frac{36 e^{j(\pi t / 6)}\left(u-2 j(\pi / 6)(j \cos t-\sin t)-u\left(\pi^{2} / 36\right)\right)}{j^{2} \pi^{2}}, \\
\mathbf{p}_{3}^{\prime}=- & -u e^{j(\pi t / 6)} \\
& -\left(216 e^{j(\pi t / 6)}(-\sin (t)+j \cos (t)+3 j u\right. \\
& -3\left(\frac{\pi^{2}}{36}\right)(j \cos t-\sin t) \\
& \left.\left.-u j\left(\frac{\pi^{3}}{216}\right)\right)\right) \times\left(j^{3} \pi^{3}\right)^{-1},
\end{aligned}
$$

where $u=\cos t+j \sin t$. If we consider the rotation angle $\varphi$ as a parameter of the motion, it is clear that the relation $\mathbf{p}_{n}^{\prime}=\mathbf{x}^{\prime}-j^{-n} \stackrel{(n)}{\mathbf{x}^{\prime}}$ holds for $n=1,2,3$.

\section{Conflict of Interests}

The authors declare that there is no conflict of interests regarding the publication of this paper.

\section{Acknowledgment}

This research has been supported by Yildiz Technical University Scientific Research Projects Coordination Department, Project no. BAPK 2013-01-03-DOP02.

\section{References}

[1] J. Keller, "Quaternionic, complex, duplex and real Clifford algebras," Advances in Applied Clifford Algebras, vol. 4, no. 1, pp. 1-12, 1994.

[2] S. Ulrych, "Representations of Clifford algebras with hyperbolic numbers," Advances in Applied Clifford Algebras, vol. 18, no. 1, pp. 93-114, 2008.

[3] A. E. Motter and M. A. Rosa, "Hyperbolic calculus," Advances in Applied Clifford Algebras, vol. 8, no. 1, pp. 109-128, 1998.

[4] P. Fjelstad, "Extending special relativity via the perplex numbers," American Journal of Physics, vol. 54, no. 5, pp. 416-422, 1986.

[5] W. Blaschke and H. R. Müller, Ebene Kinematik, Verlag Oldenbourg, München, Germany, 1956.

[6] S. Yüce and N. Kuruoğlu, "One-parameter plane hyperbolic motions," Advances in Applied Clifford Algebras, vol. 18, no. 2, pp. 279-285, 2008.

[7] G. S. Birman and K. Nomizu, "Trigonometry in Lorentzian geometry," The American Mathematical Monthly, vol. 91, no. 9, pp. 543-549, 1984.

[8] E. Nesovic and M. Petrovic-Torgasev, "Same trigonometric relation in the Lorentzian plane," Kragujevac Journal of Mathematics, vol. 25, pp. 219-225, 2003.

[9] E. Nesovic, "Hyperbolic angle function in the Lorentzian plane," Kragujevac Journal of Mathematics, vol. 28, pp. 139-144, 2005.

[10] G. Sobczyk, “The hyperbolic number plane," The College Mathematics Journal, vol. 26, p. 268, 1995. 


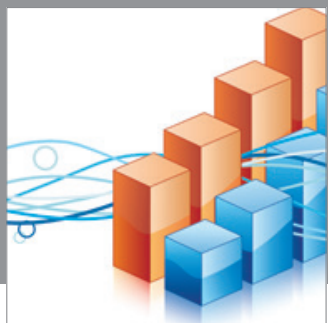

Advances in

Operations Research

mansans

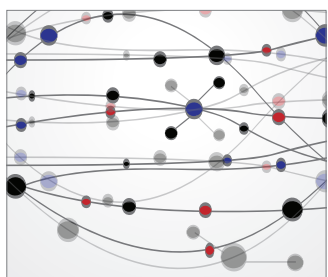

The Scientific World Journal
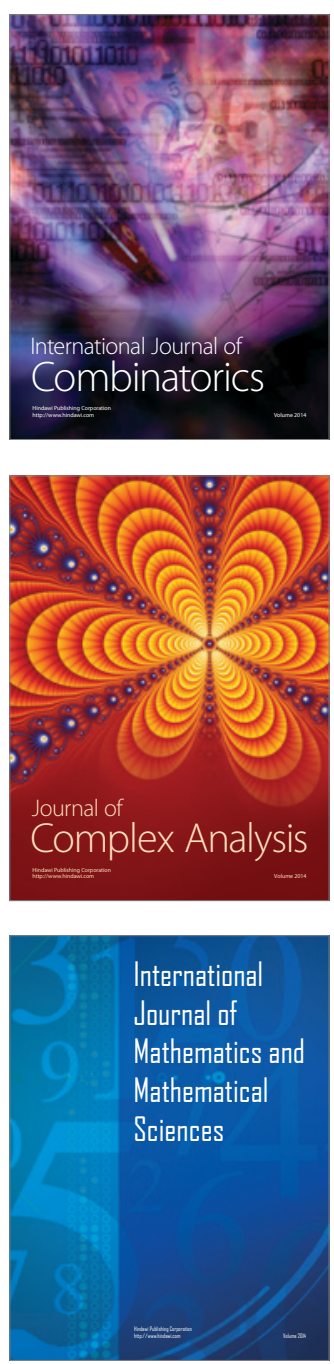
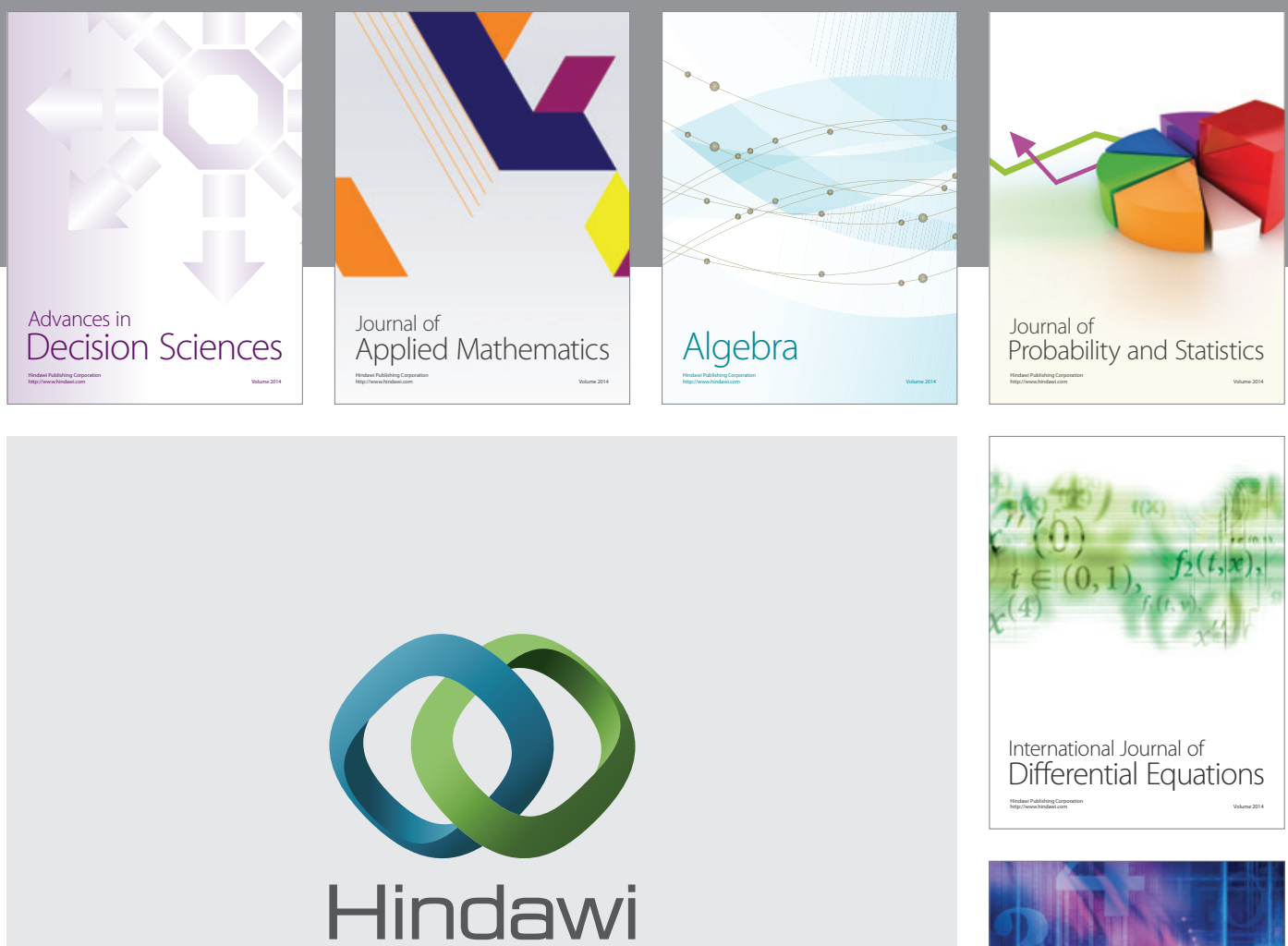

Submit your manuscripts at http://www.hindawi.com
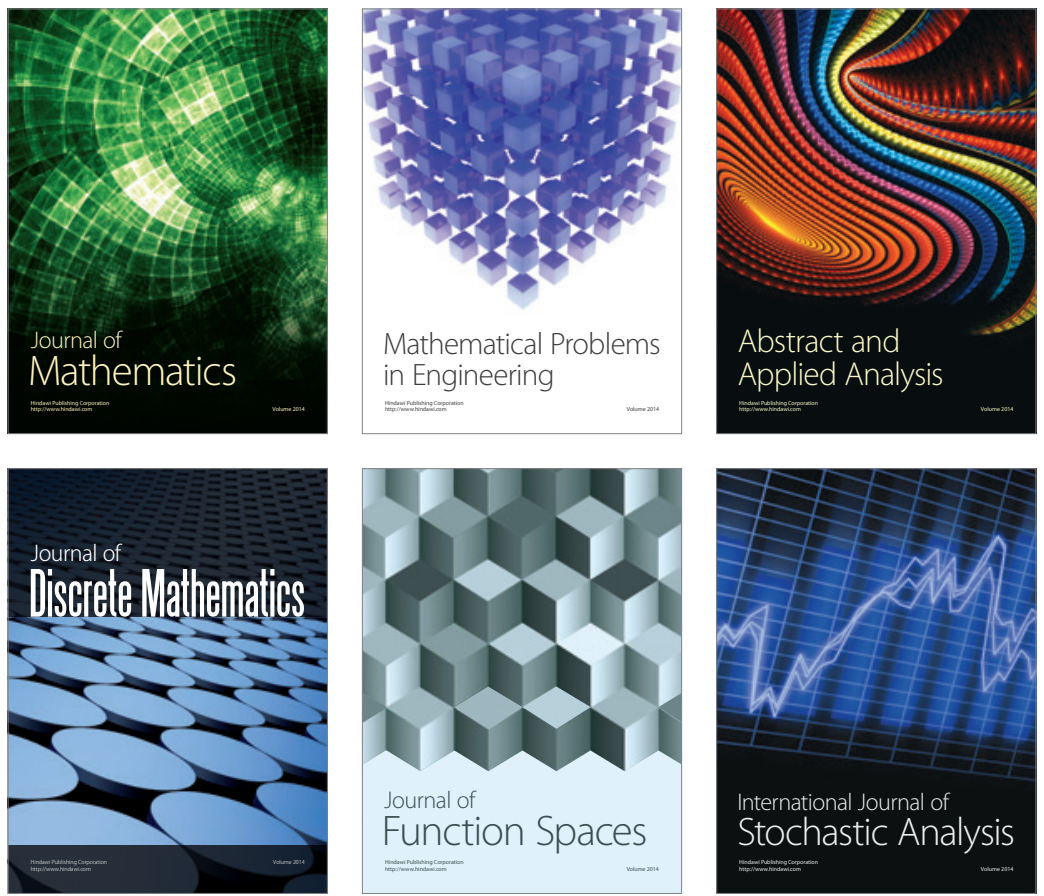

Journal of

Function Spaces

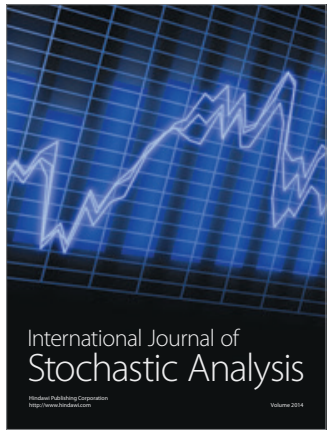

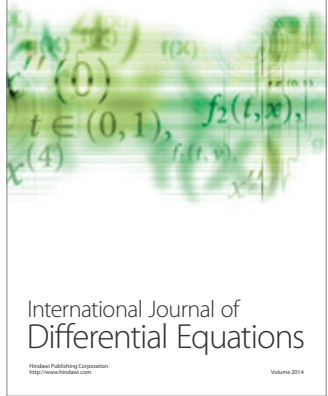
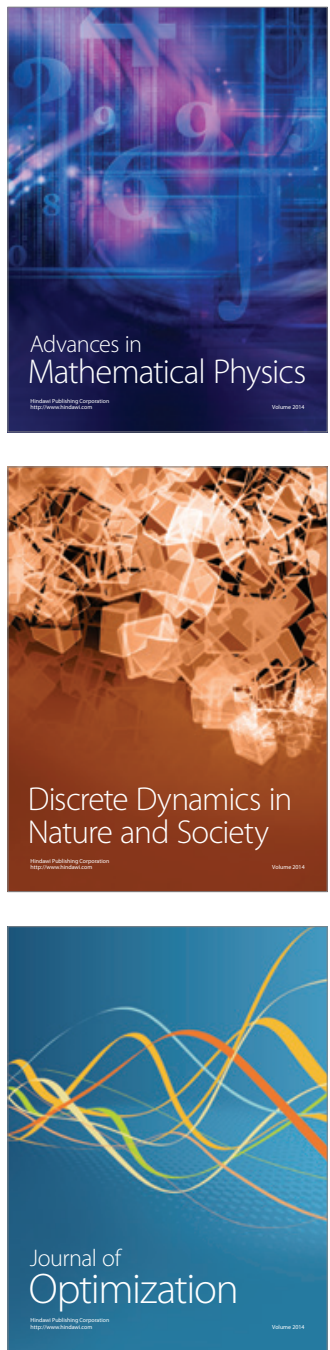